\title{
A New Method for Manufacturing Process Autonomous Planning in Intelligent Manufacturing System
}

\author{
Shuangyu Wei ${ }^{1(\bowtie)}$, Yuewei Bai ${ }^{1(\bowtie)}$, Xiaogang Wang ${ }^{1}$, Liu Kai ${ }^{1}$, \\ Lai $\mathrm{Xu}^{2}$, Paul de Vrieze ${ }^{2}$, and John Paul Kasse ${ }^{2}$ \\ 1 Shanghai Polytechnic University, Shanghai 201209, China \\ \{sywei, ywbai\}@sspu. edu.cn \\ ${ }^{2}$ Bournemouth University, Bournemouth BH12 5BB, UK
}

\begin{abstract}
This paper presents a new method for autonomous computer aided process planning (A-CAPP) in intelligent manufacturing system, in which the related input and output of the system are discussed on the basis of comparative analysis of traditional CAPP. The crucial functional components of the A-CAPP system, such as event scheduling management, manufacturing process planning, operation process/step planning, numerical control machining program planning, process simulation and evaluation, are introduced; and the methods of process knowledge management, including process feature knowledge, manufacturing resource knowledge and process method knowledge, are discussed as well. A-CAPP applied for intelligent manufacturing system can effectively support the production line reconstruction dynamically; shorten the time of production line configuration modification in accordance with customers' requirement change or market requirement fluctuation, and further more to balance the production lines load.
\end{abstract}

Keywords: Process autonomous planning · Intelligent manufacturing system • Event scheduling $\cdot$ Manufacturing process knowledge management

\section{Background}

An intelligent manufacturing system is a complex system, which involves the technologies of industrial network, industrial software, artificial intelligence, information security and others. After Germany launched the "industry 4.0 standardization roadmap" in 2014, the United States, Japan, the United Kingdom and China have also formulated their national smart manufacturing strategies. Consequently, intelligent manufacturing technology has become a research hotspot of advanced manufacturing technology.

CAPP (Computer Aided Process Planning) is one kind of indispensable industrial software in intelligent manufacturing system. It plays an important role as bridge between design and manufacturing, which determines the manufacturing process, processing operation sequence, required resources etc. [1-6]. Resources refers to the equipment, machine tools, process equipment (e.g., cutter, fixture, gauge, auxiliary appliance etc.) needed in production. According to the requirements, CAPP scheme 
needs to define (or match) the required manufacturing resources for each process operation, and then associate the relevant the technical documents with the resources, such as associate NC/CNC programs for the selected machine tools and programmed PLC programs for the automatic equipment (such as robots) etc.

In the traditional CAPP method, manufacturing experts used empirical knowledge to solve process planning problems and prepared process technical documents to guide downstream product manufacturing [7, 8]. The planning process for technical documents accounts for at least $40 \%$ to $60 \%$ of the time used in the entire production process. Currently, computer aided design (CAD), computer aided process planning (CAPP) and manufacturing (CAM) [1,9] have become indispensable industrial software in the process of modern product design and manufacturing.

\section{CAPP Development and Study Motivation}

\subsection{The State of the Art}

At present, the R\&D in process planning and production line reconstruction are mainly promoted by PTC, DASSAULT, SIEMENS and other leading companies. In addition, IBM, TOYOTA, FANUC and SAP also put forward some of relevant research and development plans recently. In terms of CAPP, various commercial CAPP systems have emerged one after another. This has laid a solid foundation for the integration of product design, process planning and production.

PTC has developed a platform, MPMLink [14]. Using the systems, enterprises can effectively support their manufacturing process management via the platform, i.e., product development, production process and manufacturing resource data of entire enterprises can all be unified managed through the system. DASSAULT has developed the DELMIA [15] digital manufacturing solution, which is based on open software architecture and provides a unified product design, process planning, and resource portfolio model (PPR) that companies can use to continuously compile and validate product processes throughout the product development process. Although this platform can closely combine the process planning and product design, it still is separated from the manufacturing resource management at the production end, and it does not fully realize the integrated management of product design, process planning and production. SIEMENS has developed Siemens PLM Software [16], which can cover product design, production planning, production engineering, production and service of five repeated iteration of industrial business process; then divide them into: Idea, Implementation and Application of three parts. The system is designed to help companies to solve the product idea generation and evolution of production and processing and optimize the three levels of change of the pattern in some extent. However, the technical preparation and production process integration still is not quite close, and it remains to be further optimized.

The popular domestic CAPP systems in China are KM CAPP, TIANHE CAPP and JINYE CAPP, among which KM CAPP is prominent and it has been successfully applied in more than 8,000 companies with hundreds of customized functional modules. However, the required input data of manufacturing resources by CAPP systems 
were static or historical. In other words, data has a large time delay and is still far away from real-time acquisition of production data. It may possibly be due to the fact that the planned process document is inapplicable for real production, especially when short production cycles are used. Presently, the research and development of autonomous CAPP system that can support enterprises to dynamically adjust production configuration, quickly make production decision in accordance with the market changes is almost non-existent.

\subsection{Study Motivation}

Process planning is the basic activity of transforming product design scheme (such as CAD model) into detailed technical documents to guide production, that is, how to establish the corresponding manufacturing process documents under the given conditions of manufacturing resources and put them into the operation site/workshop to instruct production. In this process, enterprises can shorten the delivery time to market by using effective CAPP schemes [10]. In addition, it can be used to solve basic problems such as part/component cost control, production planning and production efficiency. With the decrease of the number of experienced process engineers, the research of the advanced CAPP has gradually become a hot issue, and this situation has attracted more attention than ever before.

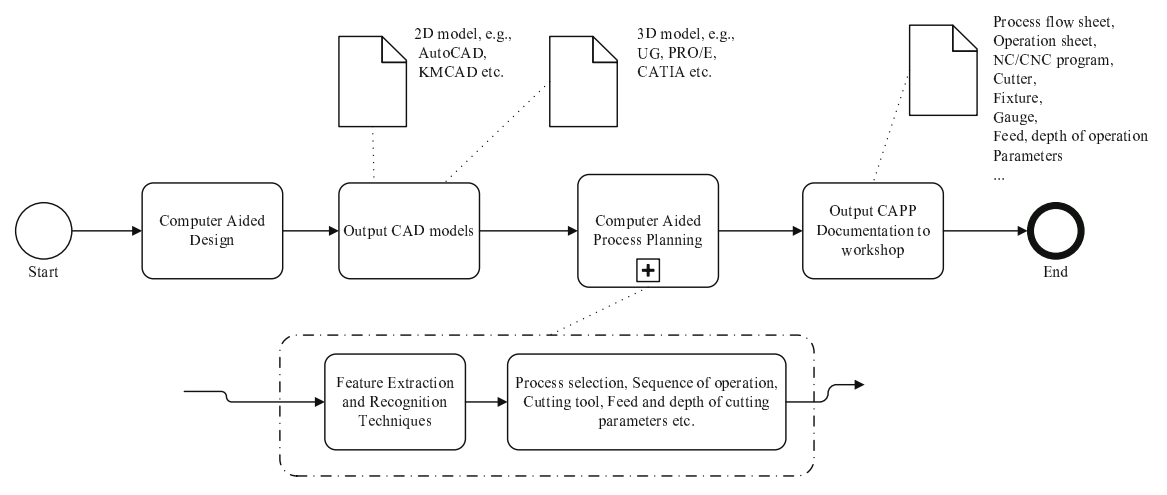

Fig. 1. The traditional CAPP planning workflow and its input

As shown in Fig. 1, the input of the CAPP mainly comes from CAD model and the manufacturing resources of workshop [4, 5]. It includes: (a) machining features; (b) geometric dimensions and tolerances of machining features (GD\&T); (c) materials of the object to be processed; (d) surface roughness of machining features; and nonCAD model information, i.e. (e) the processing capacity of the workshop, such as number of machine tools, equipment, operators, etc. CAPP can help to output the process route according to process knowledge by accurately analyzing and evaluating the input information. The final output process plan includes: (a) the selected process route; (b) defining the processing operation steps and the contents of each operation 
step; (c) selection of cutting tools; (d) selection of machining parameters; (e) design tooling, fixture etc.; (f) plan the cutting path of the tool and calculate the machining cycle to be completed; (g) estimating the manufacturing time and cost of parts.

Although many studies have been carried out on automated CAPP, the current CAPP technology is still considered to be incomplete due to the multidisciplinary characteristics and complexity of process planning [1]. And most of the current research is focused on how computer assist in process document generation, rather than autonomous generation process scheme.

A significant feature of intelligent manufacturing is to support mass customization at an economic cost. When the production line resources are abnormal, or the market feedback demand changes, or the user request to change the orders, etc., the intelligent manufacturing system can start manufacturing process autonomously planning through MES (Manufacturing Execution System), and A-CAPP will feed back the results to MES, and then realize the RMS (Reconfiguration Manufacturing System). Therefore, A-CAPP is the driving force to realize the dynamic adjustment of the production line via configuration, and RMS is the concrete implementer to realize RMS [11-13]. A-CAPP and RMS crucial parts of the intelligent manufacturing system cooperate with MES to complete the intelligent adjustment of production line resources. This paper therefore contributes to the subject with the aim to explore method of A-CAPP system development based on the heritage industrial software, and then provide a feasible reference technical solution for the relevant research.

\section{Key Technology to Build A-CAPP System}

The traditional manufacturing process planning is separated from the real-time data of the production line due to the technologies' limitation. In the process planning scheme, the resources data of the production line, e.g., machine tools, equipment, cutting tools and measuring tools, were static or historical information, which may be inconsistent with the actual resource allocation of the production line. Once the process document is released to workshop, it will guide production as a programmatic document, and the workshop must comply with. When there is a contradiction between the process plan and the actual resource usage on operation site, e.g., the production line capacity is not balanced and the bottleneck resource is seriously blocked, it will affect the actual production. When it happens, the released process document needs to be re-planned by the process engineers by following a process change workflow. It not only takes time, but also may affect the process availability after the re-planning due to the dynamic changes of the actual utilization of production resources.

A-CAPP cannot only autonomously design new process plan, but also associate the real-time data of workshop production line resource use. Among the process handling, MES will feedback the useful information to A-CAPP, which greatly improves the usability of the process schemes. In addition, it takes advantages of manufacturing process knowledge library, A-CAPP can autonomously and effectively plan the process 
sequences, defines the process operation step tasks, configures the required tools, fixtures, gauges and other process equipment for each operation step. This way, it helps to generate practical process document in line with the actual situation of the workshop manufacturing resources and can optimize reconfiguration overheads. The data processing workflow of a practical A-CAPP is described in Fig. 2.

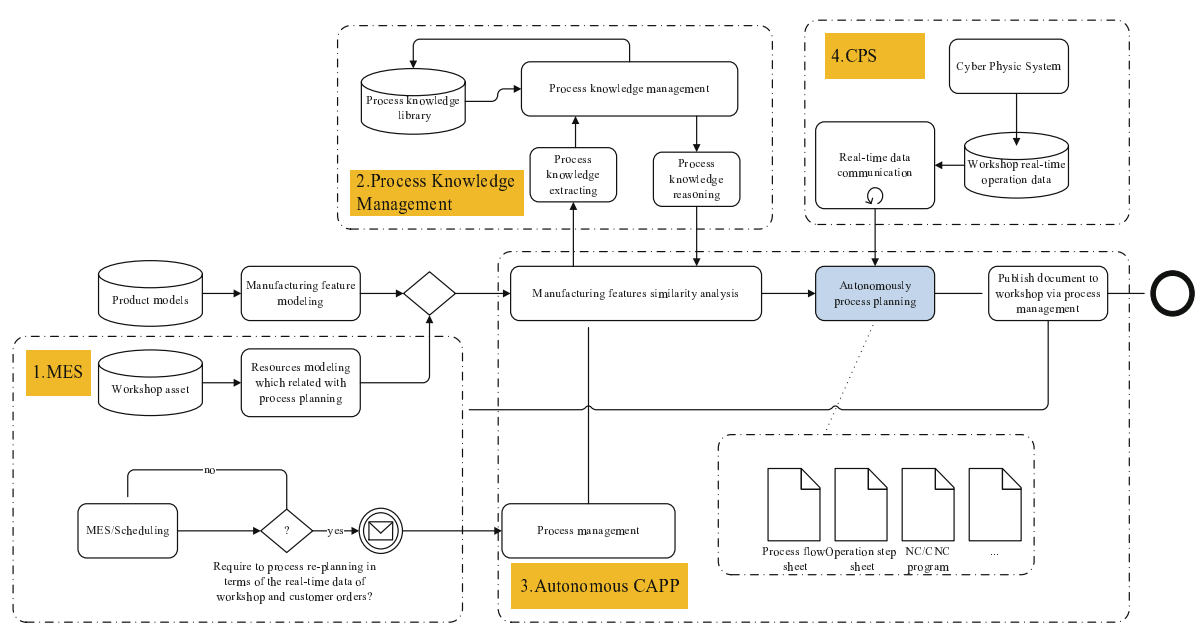

Fig. 2. Data processing workflow of A-CAPP system

\subsection{Redefining Input for A-CAPP}

Compared with the traditional CAPP, its input should be redefined properly. Ideally, it mainly comes from three sources as described in below.

(1) Firstly, it should come from MES (Manufacturing Execution System). MES can access the real-time data of production line resources, and know whether there has been a problem of uneven load of machine tools/equipment, and whether there are symptoms of task queue blocking of bottleneck resources through the CPS (cyberphysical System) and DNC (Distributed Numerical Control) System. Also MES can get the prediction information through the Business Intelligence Module through which it can predict possible adverse condition of the production line resources. When production line resources develop faults and the MES cannot adjust it via the internal job scheduling module (e.g., to balance the tasks among the machines with different $\mathrm{NC} / \mathrm{CNC}$ system), a process engineer is required to modify the pre-planned process document with the real-time data of manufacturing resources, e.g., modify process sequence, change machine tool, re-design process equipment etc. When this happens, MES can automatically trigger request 
and send message to A-CAPP; it will handle it autonomously. Because time is critical (every hour costs money) in a fault condition and autonomous manufacturing process re-planning makes it faster/cheaper.

(2) Second source input is the CPS. It collects the real-time operation data of the manufacturing resource, i.e., machine tools, equipment, robot etc., through sensor network. The data will be actively pushed to MES. MES will analyze/predict the possible abnormalities of production line resources use through the job scheduling module and manufacturing resource management module. At the same time, CPS also pushes the real-time resource data to the A-CAPP system, which is useful for relevant processes/operation steps modification. It helps to make feasible and rational modifications to the related process plans.

(3) A third source is the process knowledge management component. In the daily operation of A-CAPP, the extracted processing features, machining methods, cutting tools, fixtures, measuring tools etc., will be collected as process knowledge and stored in the process knowledge library by means of data analysis methods (e.g., clustering, classification etc.). In the late new process planning or modifying the released process plan/document, A-CAPP can deduce new process routes, operation steps, defining machine tools and equipment, tools, CNC machining programs and other information for each operation step in accordance with the processing features of the new specific mechanical part, where it will be supported in auxiliary decision-making manner by the process knowledge library. The proposed process plan set will contain multiple feasible schemes which need to be evaluated and one selected as the most feasible scheme to workshop by a process engineer. The notification can be in e-mail, SMS etc., manner sent to engineer by A-CAPP. For the changed $\mathrm{NC} / \mathrm{CNC}$ machining program, manual intervention is required, and it can only be released after an offline simulation by the process engineer. After all the changed process routes, operation steps, $\mathrm{NC} / \mathrm{CNC}$ program are evaluated, the workflow component of A-CAPP system will distributes the documents to MES system. The MES receives the new process scheme (process document, NC program, process equipment design model etc.) from A-CAPP; it will deliver and dispatch them automatically to the target machines (e.g., machine tools) by DNC and other related systems.

\subsection{The Crucial Components of A-CAPP}

The traditional CAPP mainly focused on providing tools for process document generation and design/planning of process equipment. In addition to integrating the CAPP functions, the proposed A-CAPP system needs to provide and improve some new crucial components, e.g., event manager and other components (as shown in Fig. 3), which are briefly discussed as below. 


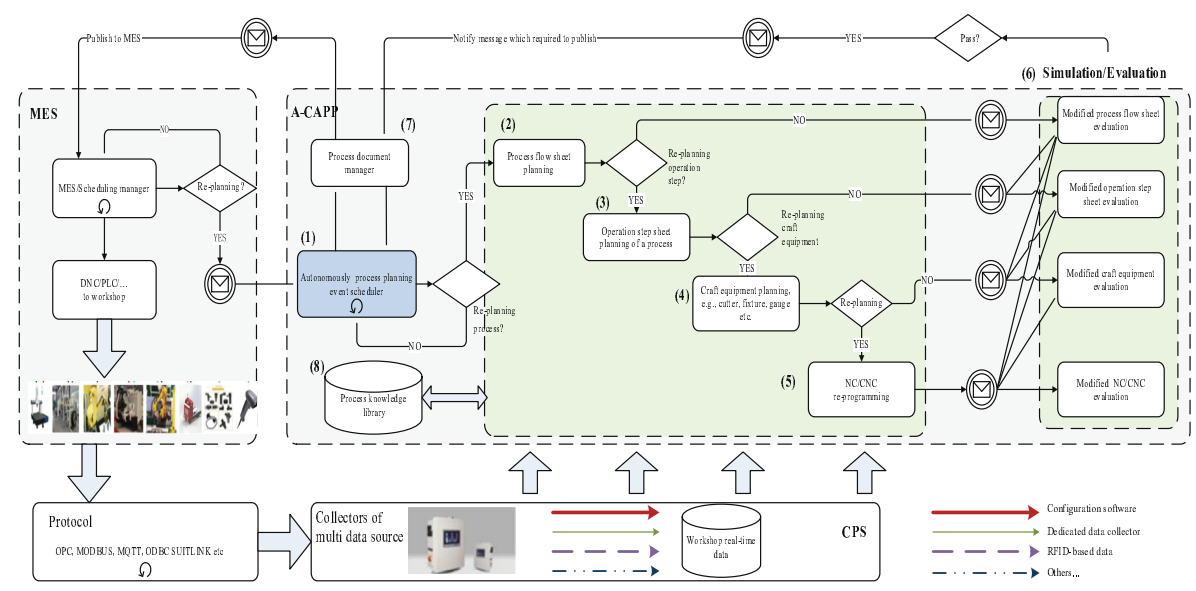

Fig. 3. The routine logic model and crucial components of A-CAPP

(1) Event manager (EM) component. It is the core component in A-CAPP implementation for autonomous planning. It is responsible for sending the start process planning command (including planning new process and modifying the release process schemes), including terminating, completing commands. For example, when the A-CAPP receives the request message with a permanent event ID (e.g., ID_ReqNumber) from MES, the A-CAPP will trigger a processing flow for the process planning. It will verify firstly through component 7 and judge whether if the request is a valid new activity. If so, the A-CAPP will handle it immediately (otherwise ignores it). When the request is confirmed to be processing, it will send a request message to component 7, which generates a new task ID_TaskNumber in line with the ID_ReqNumber. And then A-CAPP marks the activity of ID_ReqNumber as locked. It can be unlocked until the job is completed or terminated. When the A-CAPP needs to release the process document to MES, EM will send an event message of transaction request to MES by component 7 .

(2) Process flow sheet planning (PFSP) component. PFSP is a designed to make an outline scheme, which determines the process of machining, and the corresponding manufacturing resources (workstations, machine tools, equipment, type of work, etc.). It is from the overall view to describe the technical route of the part being processed. On one hand, PFSP recommends multiple process schemes based on CAD model of machining features and corresponding process knowledge; on the other hand, the proposed process schemes will comprehensively consider the factors of the real-time data of the production load, the available manufacturing resources and their processing capacity which provided by MES/DNC/CPS. In some situations, PFSP even can plan the outsourcing tasks of those the overload or overcapacity machining jobs ahead by means of the SPC data of the production lines, and then to adjust the proposed process schemes timely. As a result, the generated process document by PFSP contains a set of 
schemes, which can provide multiple feasible alternatives to workshop through manufacturing resource configuring.

(3) Operation step sheet planning component. A-CAPP is similar to traditional CAPP in operation step planning (or operation step sheet), i.e., each operation step job should be refined after the main process route is determined. First of all, it will define the items for each step that includes machining sequences and contents (geometric surfaces etc.), cutting parameters (e.g., spindle speed, cutting depth, and feed of pre revolution), the required machine tool, cutting tool, jig, measuring tools, and developing $\mathrm{NC/CNC}$ machining program according to the extracted information from CAD model (i.e., processing dimensional, tolerance, surface roughness etc.). Then to design the related cutters for operation steps in terms of the operation step sheet file.

However, the difference with the traditional CAPP is that A-CAPP develops the operation step sheet using real-time data of the production line and manufacturing resources use, for example to guide optimal resource scheduling. In the intelligent manufacturing system, A-CAPP will autonomously start modification to the released process document when the workshop organizes production accordingly but may meet problems in manufacturing resources use. MES can help to find the potential problems in resources use via CPS and DNC. When it happens, MES will work with A-CAPP to handle it accordingly in the three main way as follows:

(a) Modifying the released machining program. MES will send a request of adjusting the released process document to A-CAPP when some abnormal in manufacturing resources use are met. If the request is limited in machine tools change with different $\mathrm{NC} / \mathrm{CNC}$ (Numerical Control/Computer Numerical Control) system for a specific operation step, the related machining program (G codes) should be autonomously revised. For the modification part of machining program, it is mainly concerned with the pre-and-post processing codes due to the different requirement by varied $\mathrm{NC} / \mathrm{CNC}$ systems. However, the main part of machining codes (e.g., cutting path etc.) do not need to be modified. This also is highly frequent adjustment case that occurs in job scheduling.

(b) Modifying the released process flow sheet document. When the MES cannot solve the resources use problem by simple change machine tools, it has to modify the machining sequence (machining process route) in terms of the real-time load data of production lines. In this case, the PFSP will be triggered by A-CAPP autonomously. The PFSP will respond and handle it when receives the change request event message from EM. This is a more complicated process that will affect the related operation step sheets file.

(c) Modifying the equipment, machine tools and the other resources. When workshop encounters abnormal situation in use of equipment defined by the released process document for execution the operation step found by the MES, e.g., the planned cutters, fixtures, gauges etc. are not available, A-CAPP will be involved to fix it in terms of the real-time data of the workshop's equipment provided by MES/CPS. It will provide a few recommended change schemes (e.g., a list of the specific process equipment) for the process engineer to decide which is most suitable by using the process equipment knowledge database. 
(4) NC machining program planner component. The NC program is the control program stored in the numerical control system. It consists of numbers, words and symbols to form the machine/equipment motion control instructions, which include parameters such as cutting tool coordinate, angle and speed etc. The popular NC/CNC systems include Japanese products, e.g., FANUC (such as 16i/18i/21i), Mitsubishi (M700V, E68, etc.), MAZAK; German products, e.g., Siemens of Germany (SINUMERIK series), Heidenhain (iTNC 530), Bosch Rexroth, French Schneider (NUM1020/1040, etc.), Spanish FAGOR, Chinese Huazhong Numeral Control, etc. Different numerical control systems need different initial codes to pre-define the parameters of the systems, in order to meet the initial requirements of the coordinate system, cutting parameters and the others of the $\mathrm{NC} / \mathrm{CNC}$ system. When the modified machining program only is made due to the change of $\mathrm{NC} / \mathrm{CNC}$ system, the corresponding processing of the change (i.e., pre-and-post processing of $\mathrm{NC} / \mathrm{CNC}$ program) can be automatically processed by dedicated software kit, and then published to workshop after engineer evaluation. The other functions of NC/CNC machine program planner component are in line with the current CAM software.

(5) Process simulation and validation component. It uses computer graphics technology and dynamic programming to predict if the planned cutting path is feasible, e.g., generation free collision moving path among the designed cutter, the fixtures to hold/clamp the machining part and the other related items; and also no undercutting/overcutting situations occurring in terms of the comparison between the original $\mathrm{CAD}$ and the simulation result in advance. Process simulation functions can be realized in A-CAPP by adopting Add-In or other programming methods to integrate the third parties products of CAM simulation/verification (e.g., UG, VERICUT, Pro/E, MasterCAM, CATIA, Cimatron, EdgeCAM, CAXA, etc.), or developing CAM simulation functions. The functions of process simulation and verification are important; it belongs to the final evaluation phase before the generated process documents are released to workshop. Thus, it needs the process engineers' verification. Depending on the devices, fully automatic deployment may not be safe

(6) Process document manager component. Theoretically, the functions of process document/file manager are the part of PDM (Product Data Management). It realizes the management routines of process files version, configuration scheme, workflow (such as the flows of evaluation/audit and technical document change etc.) by associating the process document/files through the design document. Since this is concerned with releasing process documents and involving the other important technical activities, the management of the manufacturing process documents requires the intervention of the process engineers, and the process is not fully automated. Regarding the specific functions development, it can be achieved by extending the traditional PDM (Product Data Management) system and integrating the core components of A-CAPP system, such as the Event Manger (EM) component and the process knowledge library components etc. 


\subsection{Knowledge Management Component of A-CAPP}

A-CAPP can establish a reliable process knowledge library by using the knowledge of machining feature classification, manufacturing resources, machining methods (i.e., fusion of the manufacturing process and the resources). It will support autonomous planning of machining process, operation steps and developing the NC machining program, by which it can improve the overall performance of the manufacturing system, change the traditional CAPP use mode and realize the manufacturing process planning autonomously. The knowledge management component functions of A-CAPP consist of the followings.

(1) Machining feature knowledge management. This has a number of aspects. Firstly, machining feature knowledge management needs to define meta-machining features and combination methods; further more to map them with common machining features, and gradually establish the classification model of the machining features required by enterprises. Secondly, it needs to establish the mechanism of the manufacturing process information expression based on MBD (Model-based Definition), which is useful to build the association relation between the B-rep's (Boundary Representative) geometrical \& topological information of the elementary geometric objects (i.e., point, line, face of the CAD Model) and the engineering properties of the CAD model such as dimensional, tolerance, surface roughness, and the other technical requirements. The mixed expression (e.g., object-oriented semi-structured XML data format files) can be used to express the correlation model which reflects the mapping relation between the geometric features and the manufacturing features. The geometric feature recognition and machining feature mapping of the part model can be completed automatically by using the established correlation model and the results can be used as the input information of the machining features for A-CAPP.

(2) Knowledge management of machining resources. It is mainly about the information of the production line (including machine tools, equipment, robot etc.) and process equipment (such as cutting tools, fixtures, measuring tools, etc.) in the workshop, which is managed by the model of process resources. The model should have the ability to describe the resources in both static and dynamic ways that can reflect the production line, machine tool, equipment etc., from the physical structure, geometric aspect, function, information and control system of the resources. Then A-CAPP will use the information to evaluate the manufacturability of products or judge the applicable scope of the production line or equipment. The process resource model can not only support intelligent reasoning in process planning, and judge the relevance among the objects such as process features, manufacturing resources and machining methods, but also can be used to support the effectiveness analysis of process procedures.

(3) Machining process knowledge management. The machining method selection of typical process features is to judge the similarity between the current processing object and the features in the feature database by searching the pre-defined feature database, and then extract the process knowledge items with high similarity as the basic process (or template) to establish the current part process in an automatic or 
semi-automatic way. The similarity machining process analysis is realized by the clustering learning model. It needs to define the coding rules of the entire feature vector by establishing the mapping relationship between the machining process features and process plan, and then to use the learning algorithm based on the auxiliary domain data learning and fusion algorithm based on the matching model. In addition, it needs to access the quality assurance data of the machining products and the running data of the machine tools, equipment, robot etc., and the process parameters, which achieved by means of combining the actual information of the machining process features from MES with analysis result of the processing sequence, the clamping way, machining range constraint etc., plus introduction of the association rules in the learning process similarity model. Finally, it can improve the reliability of machining process similarity study performance and realize the continuous optimization for the machining parameters of the knowledge library.

(4) Process knowledge maintenance. The production process knowledge fusion model is established by means of projecting the basic information of the CAD model (e.g., geometric features, process features) and the processing resources, process parameters into the same semantic shared subspace; and then completing the data mining tasks such as association and clustering. The knowledge management component establish the relationship between the process parameters and the quality of products, equipment status data from MES, through the association data mining via the improved a priori big data association rules algorithm. Finally, it can continuously improve the effectiveness of the planned processes of parts in an autonomous way. In the process of knowledge mining, A-CAPP system need to safely share the related data across time, region, physical space and network space through the whole process of non-destructive transmission of important process data and its fusion information in encryption, compression, transmission, reception and analysis.

\section{Conclusions}

Traditional CAPP is applied in a relatively independent technical preparation phase. Although it can be integrated with collaborative design to some extent, it still occurs solely in the process of technical preparation phase and does not scale with the actual production phase. Furthermore, it used the outdated and unrealistic production data in static/historical manner, which lacks the improvement for the manufacturing process schemes and process parameters via the actual production quality data.

A-CAPP can solve or relieve it by extending and developing some crucial components, e.g., the event manager and process knowledge library and the others, based on the heritages. The autonomous process planning function can work in an integrated environment among PDM/MES/DNC/CPS in automatic and semi-automatic ways. For the important jobs of A-CAPP, e.g., valuation and review if a manufacturing process plan is feasible and whether if it can be released to workshop etc., engineer intervention is needed. The quality and the feasibility of the process schemes planned by A-CAPP will be higher than before. 
In an ideal intelligent manufacturing system, A-CAPP is able to relieve bottleneck resources constraints, balance the loads of the production lines and improve the corresponding productivity of the whole manufacturing system by using the real-time production line data provided by MES. In addition, the relationship between product quality and process parameters can be established and the product quality can be continuously optimized. Therefore, the manufacturing system with autonomous process planning ability is able to meet the needs of small batch production and personalized mass customization; and it is also the direction of the development of intelligent manufacturing.

The future work for this project will mainly focus on two aspects: one is to use the proposed method of A-CAPP based on KMCAPP, developed by KMSoft and applied widely in China, to establish a prototype system which can realize the preliminary integration with MES; the other aspect is to carry out research on dynamic reconstruction technology of production line, integrate the prototype A-CAPP system with production line configuration management system, realize the integration of A-CAPP, DNC and other related systems to reach a closed-loop control of the whole manufacturing system.

Acknowledgement. This project is funded by the State Key Research and Development Program of China (2017YFE0118700); and received funding from the European Union's Horizon 2020 research and innovation programme under the Marie Skłodowska-Curie grant agreement No 734599.

\section{References}

1. Xu, X., Wang, L., Newman, S.T.: Computer-aided process planning-a critical review of recent developments and future trends. Int. J. Comput. Integr. Manuf. 24(1), 1-31 (2011)

2. Cay, F., Chassapis, C.: An IT view on perspectives of computer aided process planning research. Comput. Ind. 34(3), 307-337 (1997)

3. Marri, H.B., Gunasekaran, A., Grieve, R.J.: Computer-aided process planning: a state of art, 261-268(1998)

4. Kumar, M., Rajotia, S.: Integration of scheduling with computer aided process planning. J. Mater. Process. Technol. 138(1-3), 297-300 (2003)

5. Bard, J.F., Feo, T.A.: The cutting path and tool selection problem in computer aided process planning. J. Manuf. Syst. 8(1), 17-26 (1989)

6. Yusof, Y., Latif, K.: Survey on computer-aided process planning. Int. J. Adv. Manuf. Technol. 75(1-4), 77-89 (2014)

7. Halevi, G., Weill, R.D.: Computer-aided process planning (CAPP). In: Halevi, G. (ed.) Principles of Process Planning, pp. 317-332. Springer, Dordrecht (1995). https://doi.org/10. 1007/978-94-011-1250-5_15

8. Ciurana, J., Ferrer, I., Gao, J.X.: Activity model and computer aided system for defining sheet metal process planning. J. Mater. Process. Technol. 173(2), 213-222 (2006)

9. Sarcar, M.M.M., Rao, K.M., Narayan, K.L.: Computer aided design and manufacturing. PHI Learning Pvt. Ltd 10. (2008)

10. Culler, D.E., Burd, W.: A framework for extending computer aided process planning to include business activities and computer aided design and manufacturing (CAD/CAM) data retrieval. Robot. Comput. Integr. Manuf. 23(3), 339-350 (2007) 
11. Rösiö, C., Säfsten, K.: Reconfigurable production system design theoretical and practical challenges. J. Manuf. Technol. Manag. 24(7), 998-1018 (2013)

12. Hasan, F., Jain, P.K., Kumar, D.: Optimum configuration selection in reconfigurable manufacturing system involving multiple part families. Opsearch 51(2), 297-311 (2014)

13. Wang, G.X., Huang, S.H., Yan, Y., Du, J.J.: Reconfiguration schemes evaluation based on preference ranking of key characteristics of reconfigurable manufacturing systems. Int. J. Adv. Manuf. Technol. 89, 2231-2249 (2017)

14. http://support.ptc.com/help/windchill/whc/whc_en/index.html\#page/Windchill_Help_Center \%2FExpMPM_Oview.html\%23. Accessed 25 Feb 2019

15. https://www.3ds.com/products-services/delmia/products/. Accessed 25 Feb 2019

16. https://www.plm.automation.siemens.com/global/en/. Accessed 25 Feb 2019 